\title{
Matrix metalloproteinase-2 plays a critical role in overload induced skeletal muscle hypertrophy
}

\author{
Qia Zhang ${ }^{1}$ \\ Sunil K Joshi ${ }^{1,3}$ \\ David H Lovett ${ }^{1,3}$ \\ Bryon Zhang ${ }^{1}$ \\ Sue Bodine ${ }^{4}$ \\ Hubert Kim ${ }^{1,2}$ \\ Xuhui Liu'1,2
}

1 San Francisco Veterans Affairs (VA) Medical Center

2 Department of Orthopaedic Surgery, University of California at San Francisco, US

3 Department of Medicine, University of California at San Francisco, US

4 Department of Physiology and Membrane Biology, University of California at Davis, US

Corresponding author:

Xuhui Liu

Department of Orthopaedic Surgery, UC San Francisco San Francisco Veterans Affairs (VA) Medical Center 1700 Owens Street,

94158 San Francisco, US

E-mail: liux@orthosurg.ucsf.edu

\section{Summary}

Background: extracellular matrix (ECM) components are instrumental in maintaining homeostasis and muscle fiber functional integrity. Skeletal muscle hypertrophy is associated with ECM remodeling. Specifically, recent studies have reported the involvement of matrix metalloproteinases (MMPs) in muscle ECM remodeling. However, the functional role of MMPs in muscle hypertrophy remains largely unknown.

Methods: in this study, we examined the role of MMP-2 in skeletal muscle hypertrophy using a previously validated method where the plantaris muscle of mice were subjected to mechanical overload due to the surgical removal of synergist muscles (gastrocnemius and soleus).

Results: following two weeks of overload, we observed a significant increase in MMP-2 activity and up-regulation of ECM components and remodeling enzymes in the plantaris muscles of wild-type mice. However, MMP-2 knockout mice developed significantly less hypertrophy and ECM remodeling in response to overload compared to their wild-type littermates. Investigation of protein synthesis rate and Akt/mTOR signaling revealed no difference between wild-type and MMP-2 knockout mice, suggesting that a difference in hypertrophy was independent of protein synthesis.

Conclusion: taken together, our results suggest that MMP-2 is a key mediator of ECM remodeling in the setting of skeletal muscle hypertrophy.

KEY WORDS: basement membrane, extracellular matrix, matrix metalloproteinase-2, skeletal muscle hypertrophy, synergistic ablation.

\section{Introduction}

Skeletal muscles possess great plasticity, which allows them to increase or decrease their size throughout adulthood, responding to both physical and chemical signals. In adult mammalian muscle, increases in muscle mass occur primarily as a result of increase in muscle fiber size (hypertrophy) instead of muscle fiber numbers (hyperplasia). The most vigorous muscle hypertrophy happens during puberty in response to an increase of anabolic hormonal signaling ${ }^{1,2}$. Increased mechanical loading, as in resistance strength training, can also induce muscle hypertrophy in adult mammals. Skeletal muscle is a well-organized tissue that is composed of myofibers and an extracellular matrix (ECM) that connects these fibers. The ECM forms a complex architecture that not only supports blood vessels and nerves, and connects myofibers, but also integrates them to ensure optimal force transmission within the muscle $e^{3-6}$. There has been a growing body of evidence showing that ECM participates in fiber force transduction, maintains normal muscle function, stimulates muscle progenitor cell differentiation, and affects a muscle's ability to adapt to disease and injury ${ }^{7,8}$. Previous studies have suggested that hypertrophic changes observed in muscle tissue are accompanied by the activation and contribution of satellite cells, which are released from the muscle fiber basement membrane and typically fuse into the preexisting muscle fibers to contribute to skeletal muscle hypertrophy ${ }^{9,10}$. However, how much these cells contribute to hypertrophy remains largely unknown.

The ECM is under vigorous remodeling during many physiological and pathological processes, including muscle growth and regeneration. Many previous studies including our own have shown that the matrix metalloproteinase (MMP) enzyme super family plays 
a principle role in skeletal muscle atrophy and ECM remodeling 11-14. A subgroup of the MMP family, gelatinases (consisting of MMP-2 and MMP-9), digests type IV collagen, the major component of basement membrane in the ECM. In a recent study, Clave et al. showed the up-regulation of MMP-2 following a hypertrophic stimulus ${ }^{15}$. However, to our knowledge, the functional role of MMP-2 following overload induced muscle hypertrophy remains undefined.

Therefore, in this study, we examine the functional role of MMP-2 in skeletal muscle hypertrophy using a functional overload, synergist ablation, model of the plantaris muscle in MMP-2 knockout and wild-type mice. We hypothesized that deletion of the MMP-2 gene will significantly impair ECM remodeling and reduce the extent of muscle hypertrophy observed.

\section{Materials and methods}

Our study meets the ethical standards of the journal ${ }^{16}$.

\section{Synergist ablation surgery}

Hypertrophy of the plantaris muscle was induced in mature (10 month old) male MMP-2 knockout (KO) mice and their wild-type (WT) littermates by functional overload, which was induced by bilateral achillotenotomy and removal of synergist muscles (gastrocnemius and soleus) according to a previously validated method ${ }^{17-19}$. Table 1 shows the number of animals used for the various characterization experiments. All procedures were approved by the San Francisco Veterans Affairs Medical Center (SFVAMC) Institutional Animal Care and Use Committee (IACUC).

\section{Muscle biomechanical testing}

All animals underwent in situ muscle mechanical testing two weeks postoperatively as previously described ${ }^{11}$. Animals were anesthetized with isoflurane and the plantaris tendon was isolated and attached to a force transducer (Grass Tech, Astro-Med Inc) using suture silk. The femur was securely clamped and fixed. A bipolar electrode was placed in the sciatic nerve to stimulate muscle contraction. Muscle maximum isometric tetanic force was measured through the PolyView16 chart recorder (Grass Tech, Astro-Med Inc) ${ }^{20}$.

\section{Muscle harvesting}

Animals were euthanized after mechanical testing. Plantaris muscles were harvested, weighed, and processed accordingly for histological or biochemical analysis (Tab. 1).

\section{Quantitative Reverse Transcription Polymerase Chain Reaction (qRT-PCR)}

To isolate total RNA, muscle samples were homogenized in $500 \mu \mathrm{L}$ of Trizol ${ }^{\circledR}$ reagent (Invitrogen Inc., Carlsbad, CA) according to the manufacturer's instructions ${ }^{21}$. Isolated RNA was quantified and normalized to synthesize cDNA using a Transcriptor First Strand cDNA Synthesis Kit (Roche Applied Bioscience Inc., Indianapolis, IN.). qRT-PCR was performed to quantify the expression of ECM components and remodeling markers using a LightCycler 480 SYBR Green I Master kit (Roche Applied Bioscience Inc.). Primer sequences are listed in Table 2. Amplification reactions were performed with 40 cycles of $\left(95^{\circ} \mathrm{C}\right.$ for $15 \mathrm{sec}$; $56^{\circ} \mathrm{C}$ for $45 \mathrm{~s}$; and $72^{\circ} \mathrm{C}$ for 1 $\mathrm{min}$ ), and normalized to GAPDH. Fold change in $\mathrm{mR}$ NA expression was calculated by using $\triangle \Delta C T^{21}$.

\section{Western blot analysis}

To isolate total protein, muscle samples were homogenized in $500 \mu \mathrm{L}$ of T-PER solution with a protease

Table 1. Experimental design and animal numbers.

\begin{tabular}{|c|c|c|c|c|}
\hline \multicolumn{5}{|c|}{ No puromycin injection ( $\mathrm{N}=48$ total) } \\
\hline & \multicolumn{2}{|c|}{ MMP-2 knockout mice } & \multicolumn{2}{|c|}{ Wild-type mice } \\
\hline & Overload & Control & Overload & Control \\
\hline $\begin{array}{l}\text { Left limb: Mechanical testing } \\
\text { Right limb: Histology }\end{array}$ & $\mathrm{N}=6$ & $\mathrm{~N}=6$ & $\mathrm{~N}=6$ & $\mathrm{~N}=6$ \\
\hline $\begin{array}{l}\text { Left limb: RNA } \\
\text { Right limb: Protein }\end{array}$ & $\mathrm{N}=6$ & $\mathrm{~N}=6$ & $\mathrm{~N}=6$ & $\mathrm{~N}=6$ \\
\hline \multicolumn{5}{|c|}{ With puromycin injection ( $\mathrm{N}=24$ total) } \\
\hline & \multicolumn{2}{|c|}{ MMP-2 knockout mice } & \multicolumn{2}{|c|}{ Wild-type mice } \\
\hline & Overload & Control & Overload & Control \\
\hline $\begin{array}{l}\text { Left \& Right : } \\
\text { Combined for SUnSET }\end{array}$ & $\mathrm{N}=6$ & $\mathrm{~N}=6$ & $\mathrm{~N}=6$ & $\mathrm{~N}=6$ \\
\hline
\end{tabular}


Table 2. Primers used for qRT-PCR. Glyceraldehyde 3-phosphate dehydrogenase (GAPDH); myogenic differentiation 1 (MyoD); myogenin; collagen, type 1, alpha 1 (Col I); collagen type IV (Col IV); fibronectin; v-laminin; matrix metalloproteinase 2 (MMP-2); matrix metalloproteinase 9 (MMP-9); matrix metalloproteinase 13 (MMP-13).

\begin{tabular}{|c|c|c|}
\hline Gene & Forward (5’ $\rightarrow 3$ ') & Reverse $\left(5^{\prime} \rightarrow 3^{\prime}\right)$ \\
\hline GAPDH & TGCACCACCAACTGCTTAG & GGATGCAGGGATGATGTTC \\
\hline MyoD & GGAAGAGTGCGGCTGTGT & GTGGAGATGCGCTCCACTAT \\
\hline Myogenin & ССTTGCTCAGСTСССТСА & TGGGAGTTGCATTCACTGG \\
\hline Col I & AAGACGGGAGGGCGAGTGCT & TCTCACCGGGCAGACCTCGG \\
\hline Col IV & CCGGGGGTTCCAGGCCCTAA & GGCACGCTCGTTGCCTTGGA \\
\hline Fibronectin & CCAAGACCTGAGCAAGGAAA & GTTCTGTGGGAGGGGTGTT \\
\hline y-Laminin & GGAGCAGAAGCGGCCTGCAA & TTCGCAGGCACCCCACCAAC \\
\hline$M M P-2$ & AGCGCAACGATGGAGGCACG & CACTTGGGCTTGCGGGGGAA \\
\hline MMP-9 & CGTGTACGGACCCGAAGCGG & AGGCAGAGTAGGAGCGGCCC \\
\hline MMP-13 & АCTCCCTGTTGGTCCCTGCCC & GGGTGCAGGCGCCAGAAGAAT \\
\hline
\end{tabular}

inhibitor cocktail (Sigma-Aldrich Inc., St. Louis, MO) as described previously ${ }^{21}$. The homogenates were centrifuged at $2,500 \times \mathrm{g}$ at $4^{\circ} \mathrm{C}$ for $10 \mathrm{~min}$. After determining the protein concentration, $20 \mu \mathrm{g}$ of protein was loaded on $10 \%$ NUPAGE Bis-Tris gels and transferred to PVDF membranes (Invitrogen Inc.) that were blocked and incubated in primary and secondary antibodies as previously described ${ }^{21}$. The following rabbit-anti-rat primary antibodies were used at a dilution of 1:500: anti-mTOR (cat\# 2972), anti-phospho-mTOR (cat\# 2971), anti-Akt (cat\# 9272), antiphospho-Akt (cat\# 9271), anti-S6K1 (cat\# 9202), antiphospho-S6K1 (cat\# 9208), and anti- $\beta$ actin (cat\# 4967). A HRP conjugated goat-anti-rabbit secondary antibody (cat\# 7074) (Cell Signaling Technology Inc., Danvers, MA) was used at a dilution of 1:10,000. Blots were developed using enhanced chemiluminescence (ECL) plus reagent (GE Healthcare Inc., Piscataway, NJ), imaged, and quantified using Image $J$ Software (NIH).

\section{Zymography}

Zymography was performed to verify activity MMP-2 using the plantaris muscle protein extract from both surgical and control groups of WT mice. Twenty microgram of protein was loaded on $10 \%$ gelatin SDSPAGE gels as described previously ${ }^{11,13}$. The intensity of MMP-2 pro-enzyme and active bands was quantified using Image-J software (NIH).

\section{In vivo SUnSET}

To measure the change of in vivo protein synthesis, we used a recently developed non radioactive technique called surface sensing of translation (SUn-
SET) ${ }^{22}$. This technique utilizes puromycin, an aminonucleoside antibiotic, that resembles a portion of an aminoacylated tRNA. Puromycin enters the A site of the ribosome from where it is transferred to the growing chain. It causes premature termination and therefore provides an indirect method of measuring global protein synthesis that can be evaluated via standard laboratory techniques such as western blotting. MMP-2 KO mice and their WT littermates from both the surgical and control groups were anesthetized and given an intraperitoneal injection of 0.04 $\mu \mathrm{mol} / \mathrm{g}$ puromycin dissolved in $1 \mathrm{~mL}$ of $\mathrm{PBS}^{22,23}$. Mice were under general anesthesia with $2 \%$ isoflurane in oxygen before and after injection. Animals were sacrificed exactly 30 minutes after injection and plantaris muscles were harvested and homogenized for western blotting as described above. PVDF membrane was probed with an anti-puromycin (3RH11, cat\# EQ0001) antibody (KeraFAST Inc., Boston, MA) at a dilution of 1:1000. After overnight incubation with the primary antibody, the blot was probed with a fluorescent IRDye $800 \mathrm{CW}$ Goat anti-Rabbit IgG secondary (cat\#926-32211) antibody (LI-COR Biosciences, Lincoln, NE) and imaged using the Odyssey Infrared Imaging System (LI-COR Biosciences) and quantified as described above. Gel loading was verified via coomassie blue staining.

\section{Total collagen staining}

Muscle samples for histological analysis were frozen in isopentane precooled in liquid nitrogen and were sectioned at $-20^{\circ} \mathrm{C}$ at a thickness of $10 \mu \mathrm{m}$. Masson's Trichrome Staining for total ECM collagen was performed for histological analysis. Fiber cross-sectional area and number were measured using Image $\mathrm{J}$ software. 


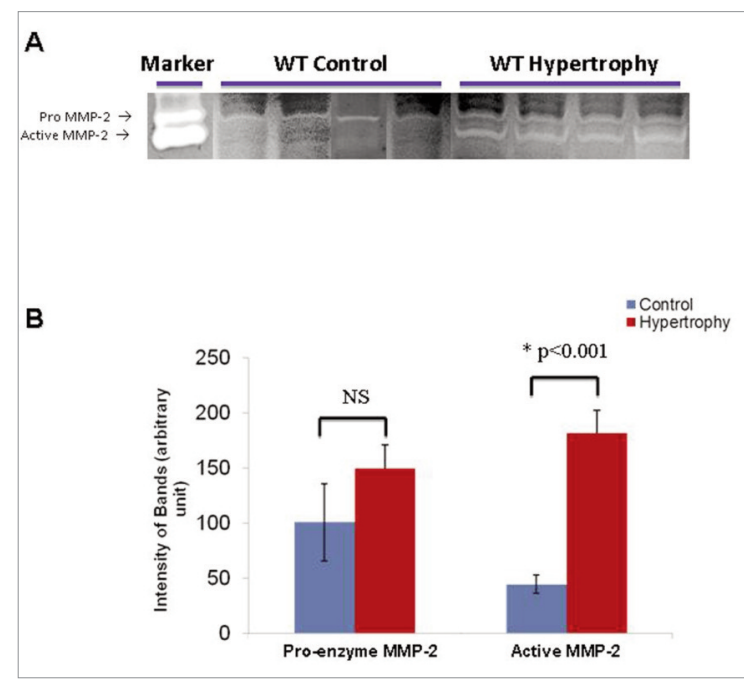

Figure 1. Zymography image showing active MMP-2 in plantaris muscle following two weeks between WT mice that underwent synergistic ablation surgery and WT control mice (A). Bands matched MMP-2 protein size and not MMP-9 (not shown). Semi-quantitative analysis of band intensity showed active form of MMP-2 was significantly upregulated in hypertrophic plantaris muscle compared to active MMP-2 in control mice (B). There was no difference in pro-enzyme MMP-2 between control and hypertrophic mice. (NS = no significant difference; * indicates significant difference).

\section{Statistical Analysis}

Two-way ANOVA with Tukey's HSD post-hoc comparison was used for data analysis to compare differences among groups (WT hypertrophy, KO hypertrophy, WT control, and KO control). A non-parametric Mann-Whitney $U$ test was used to analyze the distribution of muscle fibers. Significance was defined as a $p<0.05$. Data are presented as mean \pm standard error.

\section{Results}

\section{Increase in MMP-2 activity following overload}

Zymography showed that the active form of MMP-2 was significantly up-regulated in plantaris muscles after two weeks of hypertrophy compared to control WT mice $(p<0.001)$ (Fig. 1). The pro-enzymatic form of MMP-2 was not significantly up-regulated $(p=0.0645)$ (Fig. 1).

\section{Increase in gene expression of ECM components and MMPs following overload}

Following two weeks of overload, qRT-PCR showed that myogenic markers-myogenic differentiation 1 (MyoD) and myogenin were significantly increased in both MMP-2 WT and KO mice compared to their controls. Expression of key ECM components, including collagen type (Col I), collagen type IV (Col IV), fibronectin, and laminin, was also significantly increased following overload in both MMP-2 WT and $\mathrm{KO}$ mice compared to their controls. While MMP-9 and MMP-13 were up-regulated in both WT and KO mice following overload, MMP-2 up-regulation was seen only in WT mice. Interestingly, the up-regulation of all these genes (including MMPs) was significantly lower in MMP-2 KO mice compared to MMP-2 WT mice after overload $(\mathrm{P}<0.001)$ (Tab. 3).

Reduced increase in muscle weight and mechanical force in MMP-2 KO mice following overload

Robust hypertrophy was seen in plantaris muscles of WT mice following two weeks of overload compared to non-surgery WT controls as evident by the significant increase observed in wet weight $(p<0.001)$. However, no significant difference in muscle weight was observed be-

Table 3. ECM remodeling following overload $\left({ }^{*}, \mathrm{P}<0.001\right.$ compared to wild-type mice).

Fold Change

\begin{tabular}{lcc}
\hline & $\begin{array}{c}\text { Wild-type } \\
\text { (Fold change } \pm \text { SEM) }\end{array}$ & $\begin{array}{c}\text { MMP-2 knockout } \\
\text { (Fold change } \pm \text { SEM) }\end{array}$ \\
\hline MyoD & $54.91 \pm 7.95$ & $14.87 \pm 7.04^{*}$ \\
\hline Myogenin & $53.33 \pm 9.26$ & $23.03 \pm 3.43^{*}$ \\
\hline Col I & $56.62 \pm 0.56$ & $22.10 \pm 6.95^{*}$ \\
\hline Col IV & $65.61 \pm 12.47$ & $5.83 \pm 1.84^{*}$ \\
\hline Fibronectin & $25.62 \pm 12.19$ & $3.47 \pm 0.30 *$ \\
\hline -Laminin & $41.58 \pm 12.22$ & $14.04 \pm 1.30 *$ \\
\hline MMP-2 & $28.85 \pm 2.23$ & $\mathrm{~N} / \mathrm{A}$ \\
\hline MMP-13 & $90.57 \pm 30.39$ & $32.30 \pm 8.78 *$ \\
\hline
\end{tabular}




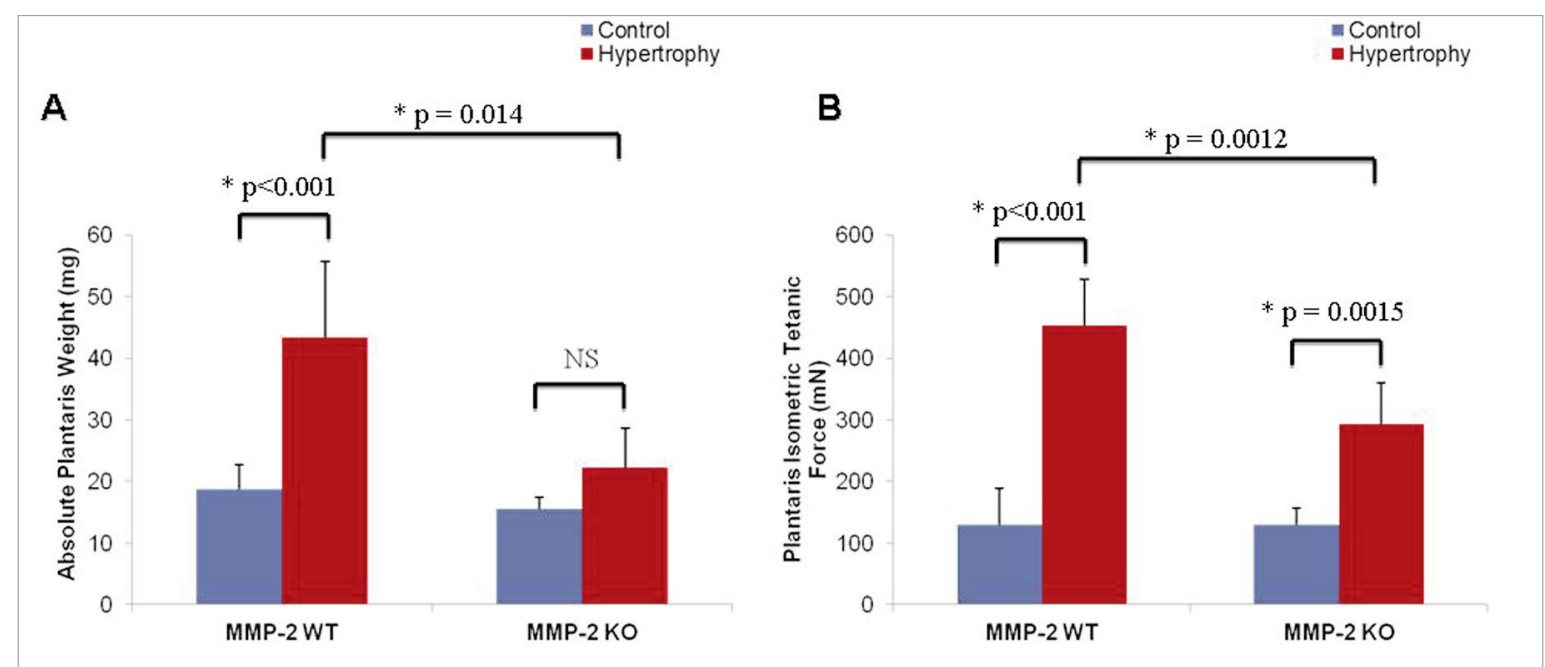

Figure 2. Muscle wet weight (A) and isometric tetanic force (B) between control and hypertrophic plantaris muscles for WT and $\mathrm{KO}$ mice. Error bars represent standard error.

(NS = not significant difference; ${ }^{*}$ indicates significant difference).

tween MMP-2 KO control mice and $\mathrm{KO}$ mice that underwent overload. In response to overload, plantaris wet weight was significantly greater in WT mice compared to KO mice $(p=0.014)$. While isometric tetanic force increased significantly in response to overload in WT and KO mice compared to their respective controls $(p<0.001$ for WT mice, $p=0.0015$ for MMP-2 KO mice), tetanic force was significantly greater in WT mice compared to KO mice following overload ( $p=0.0012)$ (Fig. 2).

\section{Reduced increase in muscle fiber number and cross-sectional area in MMP-2 KO mice following overload}

Masson's Trichrome Staining showed plantaris muscle of MMP-2 KO mice were significantly smaller than those of WT mice following overload. However, no visual differences in total collagen content were observed (Fig. 3). Muscle fiber number significantly in-

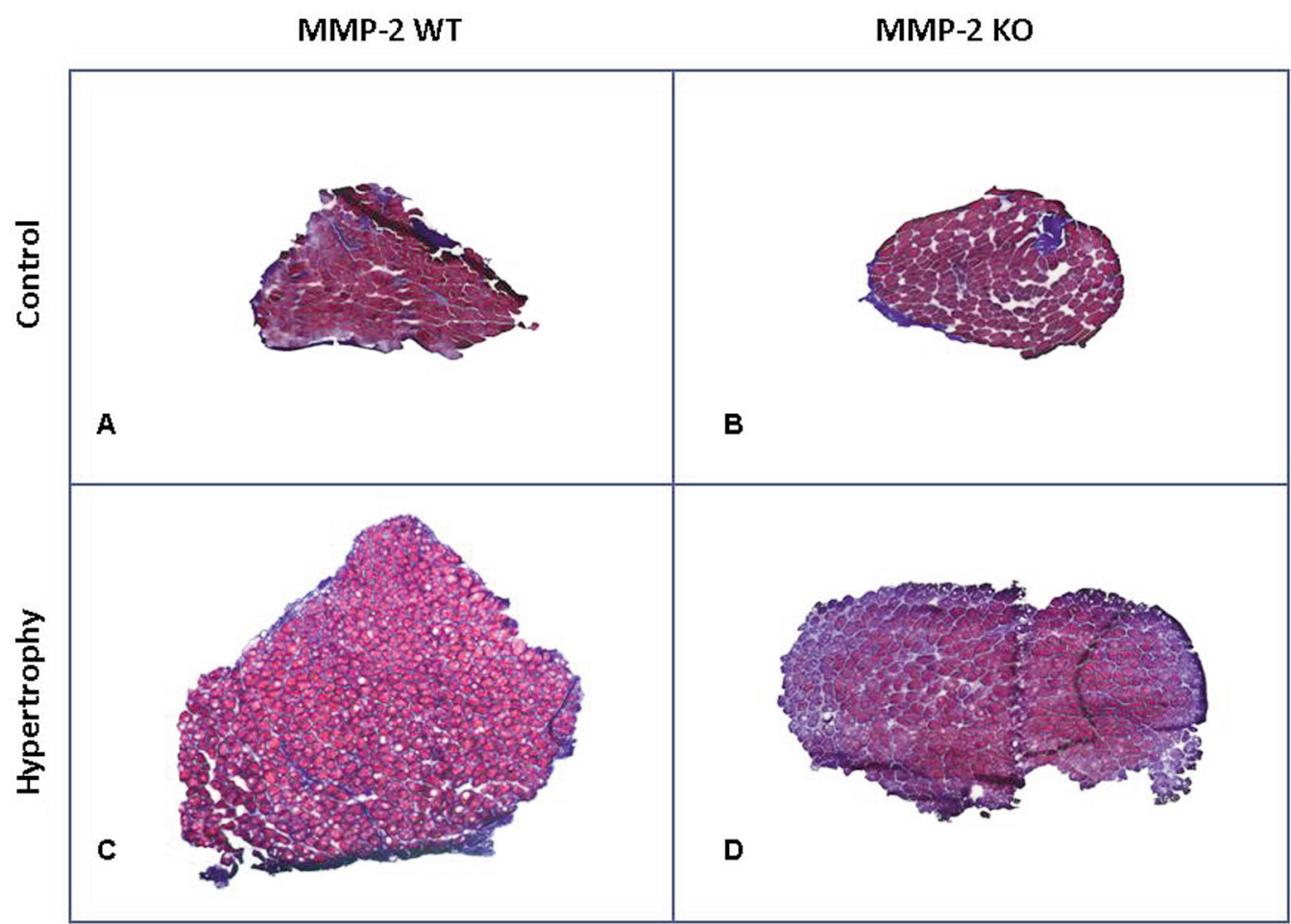

Figure 3. Images of Masson's Trichrome Staining (5x magnification) showing hypertrophic plantaris muscle of MMP-2 KO mice were significantly smaller than that of WT mice. 


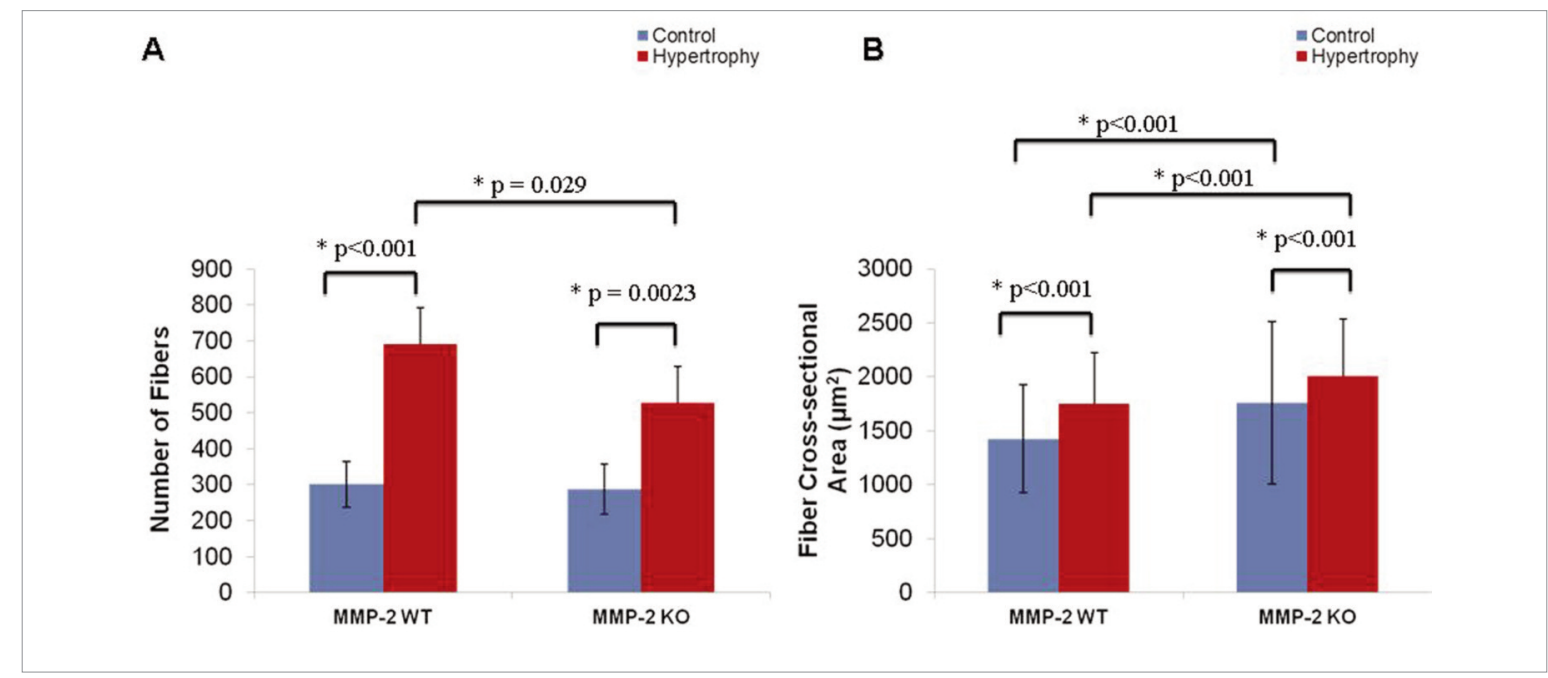

Figure 4. Muscle fiber number significantly increased in WT and MMP-2 KO mice in response to overload compared to their respective controls $(A)$. A significant increase in mean fiber cross-sectional area was seen following overload in both WT and $\mathrm{KO}$ mice $(\mathrm{B})$.

( ${ }^{*}$ indicates significant difference).

creased in WT $(p<0.001)$ and MMP-2 KO mice $(p=0.0023)$ in response to overload compared to their respective controls. However, MMP-2 KO mice had less muscle fibers compared to their WT littermate mice following overload ( $p=0.029$ ) (Fig. 4). A significant increase in mean fiber cross-sectional area was seen following overload in both WT and KO mice $(P<0.001)$. The average fiber cross-sectional area in response to overload increased $18.5 \%$ in WT mice compared to their controls (increased from $1426 \mu \mathrm{m}^{2}$ to $1750 \mu \mathrm{m}^{2}$ ). However, the average fiber cross-sectional area increased only $12.5 \%$ in MMP-2 KO mice compared to their controls (increased from $1758 \mu \mathrm{m}^{2}$ to $2011 \mu \mathrm{m}^{2}$ ) (Fig. 4).

Reduced hypertrophy in MMP-2 KO mice is not due to reduced protein synthesis

Though the rate of total protein synthesis was significantly increased in both WT and KO mice following overload compared to control mice $(p=0.0046$ for WT mice, $p=0.012$ for $K O$ mice), there was no significant differences between either MMP-2 KO and WT control groups or between MMP-2 KO and WT groups following overload (Fig. 5). We further investigated the activity of the Akt/mTOR signaling pathway, a key pathway that regulates protein synthesis, in plantaris muscles from MMP-2 KO and WT mice. Western-blot analysis of Akt, phospho-Akt, mTOR, phosphomTOR, S6K1, phospho-S6K1showed no significant difference between WT and MMP-2 KO mice following overload (Fig. 6). This suggests that MMP-2 KO mice have similar protein synthesis rate before and after overload to WT mice. Reduced plantaris muscle hypertrophy in MMP-2 KO mice was not due to changes in protein synthesis.

\section{Discussion}

Despite the importance of $\mathrm{ECM}$ remodeling in maintaining myofiber function and structural integrity, ECM remodeling during muscle hypertrophy remains under investigated. In this study, we saw active muscle ECM remodeling following overload-induced muscle hypertrophy as evident by the up-regulation of ECM components and remodeling enzymes. Deletion of the MMP-2 gene significantly decreased the extent of hypertrophy observed in the mice and, thereby suggests that MMP-2 is a pertinent contributor to muscle hypertrophy in our model. Reduced muscle hypertrophy in MMP-2 KO was most likely due to reduced muscle $\mathrm{ECM}$ remodeling rather than reduced muscle protein synthesis. Taken together, our finding suggests that MMP-2 plays a critical role in skeletal muscle hypertrophy by regulating muscle ECM remodeling.

Muscle ECM remodeling consists of both degradation of existing ECM components and synthesis of new components. Our qRT-PCR results demonstrate significant increase in gene expression of both ECM degradative enzymes including MMP-2, -9, -13, as well as key ECM components including type IV collagen, fibronectin, and $\gamma$-laminin. This data suggests that robust ECM degradation and new ECM synthesis occur simultaneously during muscle hypertrophy. Although the expression of these ECM markers was upregulated in MMP-2 KO mice following overload, the fold change of these genes was significantly lower than their WT littermates. Deletion of the MMP-2 gene significantly hampers the up-regulation of key ECM components and remodeling enzymes. This data suggests that MMP-2 may serve as a central regulator of ECM remodeling in the setting of muscle hypertrophy. 


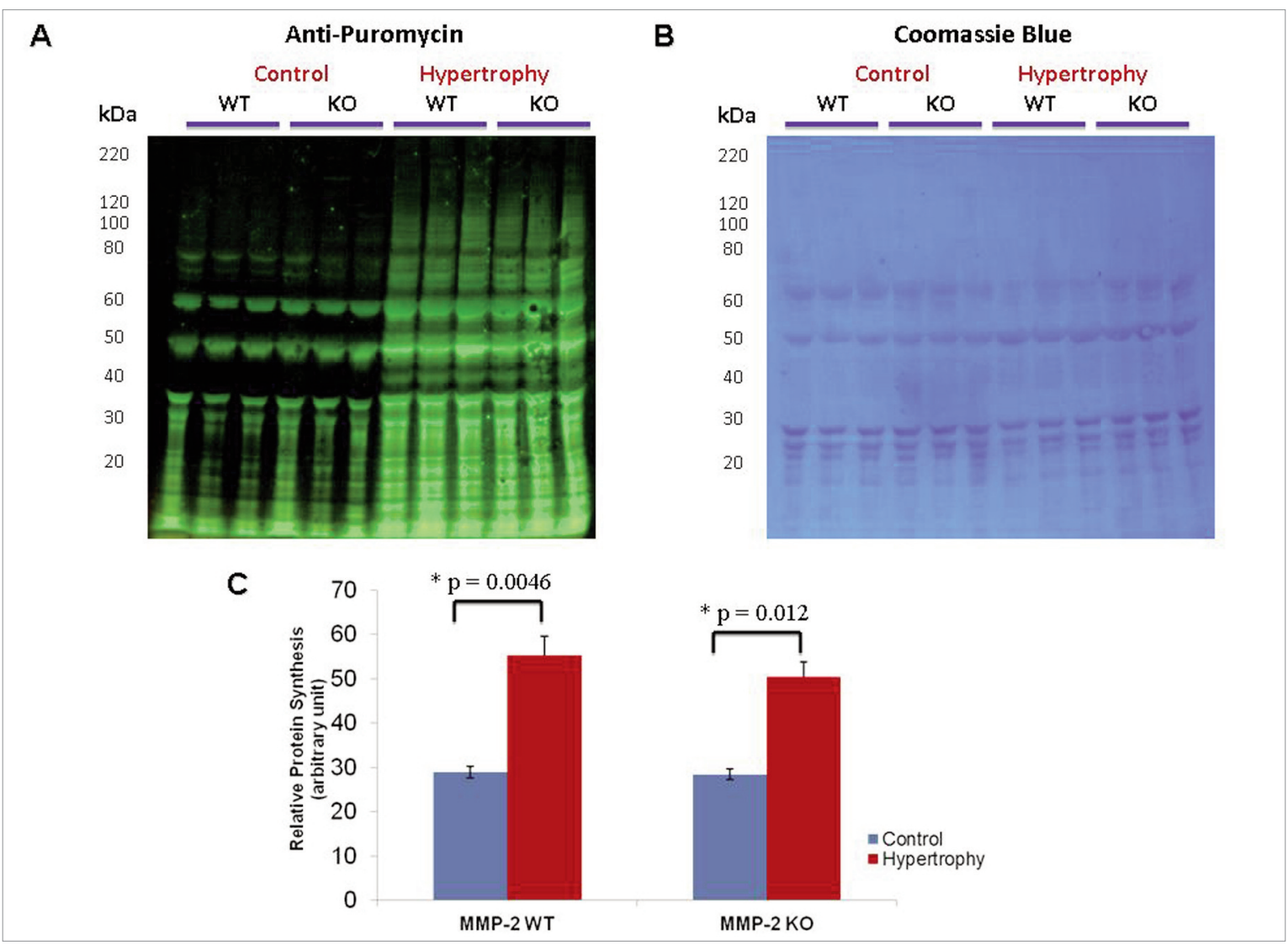

Figure 5. Typical image of Western Blot analysis for puromycin (A) followed by Coomassie Blue staining (B) to verify equal loading. Semi-quantitative measurement of band intensity (C) showed higher global protein activity following 2 weeks of plantaris hypertrophy. However, no significant difference was found between MMP-2 KO and WT mice in either control or hypertrophy group.

(* indicates significant difference).

MMP-2 is an enzyme that is involved in basement membrane remodeling. Type IV collagen is a known substrate of MMP-2, which undergoes elevated expression in various physiological and pathological events related to basement membrane metabolism, such as tissue repair, angiogenesis, and arterial enlargement ${ }^{12,24-27}$. In our preliminary data (not presented), we observed significantly increased MMP-2 activity and degradation of type IV collagen in WT mice following overload. However, type IV collagen was well preserved in MMP-2 KO mice following overload. This dichotomy in degradation of type IV collagen suggests that MMP-2 plays a dominant role in basement membrane degradation following skeletal muscle hypertrophy. However, to confirm our staining an antibody that specifically binds to fragmented type IV collagen is needed.

MyoD and myogenin are key transcription factors responsible for muscle progenitor cell myogenesis. Previous studies have shown that these transcription factors play key roles in muscle hypertrophy ${ }^{28,29}$. In line with these studies, we observed significantly increased expression of these two genes in plantaris muscles following overload, thereby further indicating the role of these two transcription factors in muscle hypertrophy. The expression level of these two genes was significantly lower in hypertrophic muscles of MMP-2 KO mice compared to WT mice. This is consistent with the reduced muscle hypertrophy observed in MMP-2 KO mice.

In order to verify that decreased ECM remodeling as opposed to decreased protein synthesis resulted in decreased hypertrophy in MMP-2 KO mice, we assessed total protein synthesis rate and a key protein synthesis pathway, the Akt/mTOR signaling pathway $^{30}$. Our evaluation of protein synthesis shows that the difference in hypertrophy we observed between WT and KO MMP-2 mice is unrelated to differences in protein synthesis rate. Likewise, no difference in phosphorylation was seen for Akt, mTOR, and S6K1 between wild-type and KO hypertrophied mice. These results further support that reduced hypertrophy seen in MMP-2 KO mice was due to reduced ECM remodeling rather than changes in protein synthesis and/or phosphorylation.

Although our study revealed the critical role of MMP-2 in skeletal muscle hypertrophy, there are several limitations to this study. We only assessed hypertrophy 


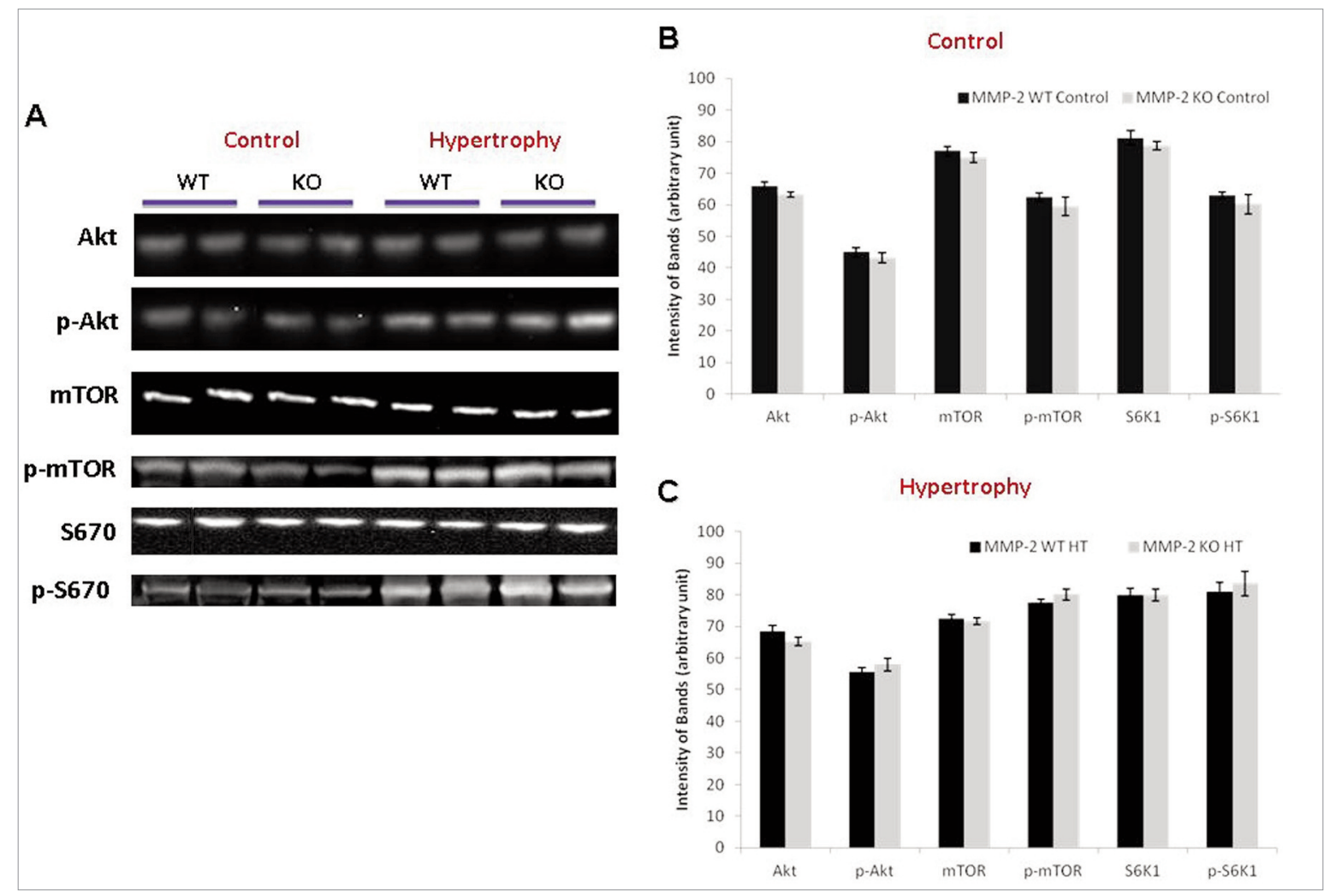

Figure 6. Typical images of Western Blot bands for Akt, mTOR, S6K1, and their phosphorylated forms in control and hypertrophic MMP-2 KO and WT mice (A). Semi-quantitative measurement of band intensity showed equal protein activity between MMP-2 WT and KO mice in either control (B) or hypertrophy group (C).

with a single time-point of two weeks. However, many studies have shown that two weeks is an appropriate time point to assess plantaris muscle hypertrophy following synergist ablation surgery ${ }^{13,31}$. In addition to these studies, in this study, we saw a robust hypertrophic response following two weeks in plantaris muscles and, therefore we believe that two weeks was an appropriate time point for assessment of hypertrophy. Future studies will evaluate hypertrophy following longer time-points. Secondly, no sham surgery was performed in the control group. Therefore, any inflammation induced by surgery was unaccounted. Since we are comparing the MMP-2 KO and WT mice in surgery and control groups separately, the effects of inflammation should not affect the overall conclusion. Third, only one hypertrophy model (functional overload) was used in this study. Other hypertrophy models, like exercise-induced hypertrophy will be considered for a future study. Finally, the expression and activity of internal MMP-2 activator and inhibitors (like MMP-14 and TIMPs) were not measured in this study. Future work is needed to establish the MMP-2 regulating network in skeletal muscle hypertrophy.

In summary, we report that deletion of the MMP-2 gene in mice significantly reduces muscle hypertrophy compared to their KO littermates following over- load. Our finding suggests that MMP-2 plays a critical role in regulating muscle size and contractility in the setting of muscle hypertrophy via regulating muscle ECM remodeling.

\section{Acknowledgements}

Qia Zhang and Sunil K. Joshi have equally contributed to this work.

This work was supported by the VA RR\&D Merit Review Grant (RX000195). The authors thank Dr. Rajabrata Sarkar (University of Maryland) for his kind gift of MMP-2 knockout mice.

\section{References}

1. Aggarwal $T$, Polanco MJ, Scaramuzzino $C$, et al. Androgens affect muscle, motor neuron, and survival in a mouse model of SOD1-related amyotrophic lateral sclerosis. Neurobiology of aging. 2014.

2. Dubois V, Laurent MR, Sinnesael M, et al. A satellite cell-specific knockout of the androgen receptor reveals myostatin as a direct androgen target in skeletal muscle. FASEB journal: official publication of the Federation of American Societies for Experimental Biology. 2014.

3. Cukierman E, Pankov R, Stevens DR, Yamada KM. Taking 
cell-matrix adhesions to the third dimension. Science (New York, NY). 2001;294(5547):1708-1712.

4. Kjaer M. Role of extracellular matrix in adaptation of tendon and skeletal muscle to mechanical loading. Physiological reviews. 2004;84(2):649-698.

5. Grazi E. Experimental basis of the hypotheses on the mechanism of skeletal muscle contraction. Muscles, ligaments and tendons journal. 2011;1(3):77-84.

6. Maas $\mathrm{H}$, Huijing PA. Myofascial force transmission between transferred rat flexor carpi ulnaris muscle and former synergistic palmaris longus muscle. Muscles, ligaments and tendons journal. 2011;1(4):127-133.

7. Gillies AR, Lieber RL. Structure and function of the skeletal muscle extracellular matrix. Muscle \& nerve. 2011;44(3):318 331.

8. Ohlendieck K. Proteomic profiling of skeletal muscle plasticity Muscles, ligaments and tendons journal. 2011;1(4):119-126.

9. Yamada M, Sankoda Y, Tatsumi R, et al. Matrix metalloproteinase-2 mediates stretch-induced activation of skeletal muscle satellite cells in a nitric oxide-dependent manner. The international journal of biochemistry \& cell biology. 2008;40(10): 2183-2191.

10. Yamada M, Tatsumi R, Kikuiri T, et al. Matrix metalloproteinases are involved in mechanical stretch-induced activation of skeletal muscle satellite cells. Muscle \& nerve. 2006;34(3): 313-319.

11. Zhang Q, Joshi SK, Manzano G, Lovett DH, Kim HT, Liu X Original article Muscle extracellular matrix degradation and contractibility following tendon rupture and disuse. Muscles, ligaments and tendons journal. 2013;3(1):35-41.

12. Liu X, Lee DJ, Skittone LK, Natsuhara K, Kim HT. Role of gelatinases in disuse-induced skeletal muscle atrophy. Muscle \& nerve. 2010;41(2):174-178.

13. Skittone LK, Liu X, Tseng A, Kim HT. Matrix metalloproteinase-2 expression and promoter/enhancer activity in skeletal muscle atrophy. Journal of orthopaedic research: official publication of the Orthopaedic Research Society. 2008;26(3): 357-363.

14. Giannelli G, De Marzo A, Marinosci F, Antonaci S. Matrix metalloproteinase imbalance in muscle disuse atrophy. Histology and histopathology. 2005;20(1):99-106.

15. Calve S, Isaac J, Gumucio JP, Mendias CL. Hyaluronic acid, HAS1, and HAS2 are significantly upregulated during muscle hypertrophy. American journal of physiology Cell physiology. 2012;303(5):C577-588.

16. Padulo J, Oliva F, Frizziero A, Maffulli N. Muscles, Ligaments and Tendons Journal. Basic principles and recommendations in clinical and field science research. MLTJ. 2013;4: 250-252.

17. Bodine SC, Baar K. Analysis of skeletal muscle hypertrophy in models of increased loading. Methods in molecular biology (Clifton, NJ). 2012;798:213-229.
18. McCarthy JJ, Mula J, Miyazaki M, et al. Effective fiber hypertrophy in satellite cell-depleted skeletal muscle. Development (Cambridge, England). 2011;138(17):3657-3666.

19. Jackson JR, Mula J, Kirby TJ, et al. Satellite cell depletion does not inhibit adult skeletal muscle regrowth following unloadinginduced atrophy. American journal of physiology Cell physiology. 2012;303(8):C854-861.

20. Jin J, Limburg S, Joshi SK, et al. Peripheral nerve repair in rats using composite hydrogel-filled aligned nanofiber conduits with incorporated nerve growth factor. Tissue engineering Part A. 2013;19(19-20):2138-2146.

21. Liu X, Joshi SK, Samagh SP, et al. Evaluation of Akt/mTOR activity in muscle atrophy after rotator cuff tears in a rat model. Journal of orthopaedic research: official publication of the Orthopaedic Research Society. 2012;30(9):1440-1446.

22. Goodman CA, Hornberger TA. Measuring protein synthesis with SUnSET: a valid alternative to traditional techniques? Exercise and sport sciences reviews. 2013;41(2):107-115.

23. Joshi SK, Kim HT, Feeley BT, Liu X. Differential ubiquitin-proteasome and autophagy signaling following rotator cuff tears and suprascapular nerve injury. Journal of orthopaedic research : official publication of the Orthopaedic Research Society. 2014;32(1):138-144.

24. Westermarck J, Kahari VM. Regulation of matrix metalloproteinase expression in tumor invasion. FASEB journal: official publication of the Federation of American Societies for Experimental Biology. 1999;13(8):781-792.

25. Buonocore D, Rucci S, Vandoni M, Negro M, Marzatico F. Oxidative system in aged skeletal muscle. Muscles, ligaments and tendons journal. 2011;1(3):85-90.

26. Hudlicka O. Microcirculation in skeletal muscle. Muscles, ligaments and tendons journal. 2011;1(1):3-11.

27. Rizzi R, Bearzi C, Mauretti A, Bernardini S, Cannata S, Gargioli $C$. Tissue engineering for skeletal muscle regeneration. Muscles, ligaments and tendons journal. 2012;2(3):230234.

28. Aguiar AF, Vechetti-Junior IJ, Alves de Souza RW, et al. Myogenin, MyoD and IGF-I regulate muscle mass but not fibertype conversion during resistance training in rats. International journal of sports medicine. 2013;34(4):293-301.

29. Zanou N, Gailly P. Skeletal muscle hypertrophy and regeneration: interplay between the myogenic regulatory factors (MRFs) and insulin-like growth factors (IGFs) pathways. Cellular and molecular life sciences: CMLS. 2013;70(21):41174130.

30. Hwee DT, Baehr LM, Philp A, Baar K, Bodine SC. Maintenance of muscle mass and load-induced growth in Muscle RING Finger 1 null mice with age. Aging cell. 2014;13(1):92101.

31. Elder GC, McComas AJ. Development of rat muscle during short- and long-term hindlimb suspension. Journal of applied physiology (Bethesda, Md: 1985). 1987;62(5):1917-1923. 